\title{
Effects of genomic selection on genetic improvement, inbreeding, and merit of young versus proven bulls
}

\author{
A. P. W. de Roos, ${ }^{\dagger} \dagger^{1}$ C. Schrooten, ${ }^{\star}$ R. F. Veerkamp, $\neq$ and J. A. M. van Arendonk $\dagger$ \\ ${ }^{*}$ CRV, PO Box 454, 6800 AL Arnhem, the Netherlands \\ †Animal Breeding and Genomics Centre, Wageningen University, PO Box 338, $6700 \mathrm{AH}$ Wageningen, the Netherlands \\ $\ddagger$ Animal Breeding and Genomics Centre, Wageningen Livestock Research Institute, PO Box 65, 8200 AB Lelystad, the Netherlands
}

\section{ABSTRACT}

Genomic selection has the potential to revolutionize dairy cattle breeding because young animals can be accurately selected as parents, leading to a much shorter generation interval and higher rates of genetic gain. The aims of this study were to assess the effects of genomic selection and reduction of the generation interval on the rate of genetic gain and rate of inbreeding. Furthermore, the merit of proven bulls relative to young bulls was studied. This is important for breeding organizations as it determines the relative importance of progeny testing. A closed nucleus breeding scheme was simulated in which 1,000 males and 1,000 females were born annually, 200 bulls were progeny tested, and 20 sires and 200 dams were selected to produce the next generation. In the "proven" (PROV) scenario, only cows with own performance records and progenytested bulls were selected as parents. The proportion of the genetic variance that was explained by simulated marker information $(M)$ was varied from 0 to $100 \%$. When $M$ increased from 0 to $100 \%$, the rate of genetic gain increased from 0.238 to 0.309 genetic standard deviations $(\sigma)$ per year $(+30 \%)$, whereas the rate of inbreeding reduced from 1.00 to $0.42 \%$ per generation. Alternatively, when young cows and bulls were selected as parents (YNG scenario), the rate of genetic gain for $M=0 \%$ was $0.292 \sigma / \mathrm{yr}$ but the corresponding rate of inbreeding increased substantially to $3.15 \%$ per generation. A realistic genomic selection scheme (YNG with $M$ $=40 \%)$ gave $108 \%$ higher rate of genetic gain $(0.495 \sigma /$ yr) and approximately the same rate of inbreeding per generation as the conventional system without genomic selection (PROV with $M=0 \%$ ). The rate of inbreeding per year, however, increased from 0.18 to $0.52 \%$ because the generation interval in the YNG scheme was much shorter. Progeny-testing fewer bulls reduced the rate of genetic gain and increased the rate of inbreeding

Received April 20, 2010.

Accepted November 24, 2010

${ }^{1}$ Corresponding author: sander.de.roos@crv4all.com for PROV, but had negligible effects for YNG because almost all sires were young bulls. In scenario YNG with $M=40 \%$, the best young bulls were superior to the best proven bulls by $1.27 \sigma$ difference in genomic estimated breeding value. This superiority increased even further when fewer bulls were progeny tested. This stochastic simulation study shows that genomic selection in combination with a severe reduction in the generation interval can double the rate of genetic gain at the same rate of inbreeding per generation, but with a higher rate of inbreeding per year. The number of progeny-tested bulls can be greatly reduced, although this will slightly affect the quality of the proven bull team. Therefore, it is important for breeding organizations to predict the future demand for proven bull semen in light of the increasing superiority of young bulls.

Key words: genomic selection, breeding program, genetic gain, inbreeding

\section{INTRODUCTION}

Genomic selection refers to genetic improvement of animals or plants through selection based on genomic breeding values (GEBV). Genomic breeding values are computed using a reference population of animals that have high density genotype as well as phenotypic information (Meuwissen et al., 2001). Genomic selection may result in higher rates of genetic gain over traditional selection using BLUP EBV because GEBV have higher reliabilities than BLUP EBV, especially for young animals, and because young animals with high GEBV become attractive to be selected as parents, which reduces the generation interval (Schaeffer, 2006). Furthermore, genomic selection can be used to decrease the rate of inbreeding because Mendelian sampling effects can be estimated more accurately, which reduces the co-selection of relatives (Daetwyler et al., 2007).

Genetic improvement programs in many animal and plant species will benefit from application of genomic selection. The advantages may be greatest for dairy cattle breeding programs because the generation interval in traditional progeny-testing schemes is long and 
selection of young bulls for progeny testing is inaccurate (Schaeffer, 2006). Furthermore, thousands of bulls that have been progeny tested in the last decades are available as a reference population with very reliable phenotypes, leading to GEBV with high reliabilities (VanRaden et al., 2009). For these reasons, the uptake of genomic selection in dairy cattle in recent years has been very high, with thousands of reference bulls and selection candidates being genotyped in many countries, and substantial research and development resources going into GEBV estimation (Hayes et al., 2009).

The use of marker-assisted selection in dairy cattle breeding programs was studied widely before the introduction of genomic selection (e.g., Smith, 1967; Soller and Beckmann, 1983; Kashi et al., 1990; Meuwissen and van Arendonk, 1992; Mackinnon and Georges, 1998; Spelman et al., 1999; Schrooten et al., 2005). These studies have shown that marker-assisted preselection of young bulls before progeny testing can increase the rate of genetic gain by 20 to 30\% (Kashi et al., 1990; Schrooten et al., 2005), but much higher gains can be reached if also young animals without their own or their progeny's performance information are used as parents for the next generation (Meuwissen and van Arendonk, 1992; Spelman et al., 1999; Schrooten et al., 2005).

Before dramatic changes to breeding programs are made, some issues need further attention. First, the rate of inbreeding in genomic selection schemes is expected to be lower than in traditional BLUP selection schemes because Mendelian sampling effects can be estimated more accurately for young animals, which may lead to less co-selection of sibs (Daetwyler et al., 2007). If we consider the use of young bulls as sires as opposed to proven bulls, however, the estimates of Mendelian sampling effects are in fact less accurate. As a result, truncation selection of young sires on GEBV will lead to more co-selection of relatives and therefore more inbreeding. Selection of young bulls before progeny testing, however, also involves co-selection of relatives. Therefore, it is difficult to predict how transitioning a progeny-test scheme to a genomic selection scheme will affect the rate of inbreeding. Hence, the effects of applying genomic selection and using young animals as parents on the rate of inbreeding need further study.

Second, dairy cattle breeding organizations need to answer the question of how many bulls still need to be progeny tested. Because of the high costs of progeny testing, there has been a tendency to reduce the number of progeny-tested bulls, but this may decrease the competitiveness of the resulting proven bull team. With higher reliabilities of GEBV and higher rates of genetic gain, relatively more young bulls will be used as sires, and proven bulls will have less influence on the rate of genetic gain. If proven bulls do not influence the rate of genetic gain, they may not need to be progeny tested. Another reason to progeny-test bulls, however, is because some farmers may prefer to use proven bulls rather than young bulls because of their higher reliability, even if their GEBV are slightly lower. The future market share of proven bull semen versus young bull semen, however, needs to be predicted accurately to determine how many bulls should be progeny tested. This market share depends on the difference in GEBV between the highest proven and young bulls. Therefore, how proven bulls will rank relative to young bulls needs to be studied.

The first objective of this study was to assess the effects of reliability of GEBV and the use of young animals as parents on the rate of genetic gain and the rate of inbreeding. The second objective was to study the merit of proven bulls versus young bulls, with different numbers of bulls progeny tested.

\section{MATERIALS AND METHODS}

\section{Breeding Program Design}

A closed nucleus breeding program was simulated in which 1,000 females and 1,000 males were born annually (Table 1). All 1,000 females received a performance phenotype when they were $3 \mathrm{yr}$ old, and all females were culled when they were 6 yr old. Of the 1,000 bulls born annually, the 200 bulls with the highest GEBV were progeny tested outside the nucleus and were culled when they were 8 yr old. The remaining 800 bulls per year were culled directly after birth. Bulls that were progeny tested obtained a phenotypic record when they were $5 \mathrm{yr}$ old. The GEBV were estimated from genomic information, phenotypes, and pedigree, as described later. Each year, the 200 highest GEBV cows of at least $3 \mathrm{yr}$ old and the 20 highest GEBV bulls of at least $5 \mathrm{yr}$ old were selected as parents to produce the next generation. It was assumed that selected dams were flushed to produce embryos, and oocytes were harvested and fertilized in vitro using multiple sires. Each dam produced 10 progeny and each sire produced 100 progeny. Sires were randomly mated to dams to produce 1 progeny per mating, so by chance some animals may have full sibs. The breeding scheme was run for 50 yr. Genomic selection was introduced in year 26 , which had several consequences (Table 2): (1) genomic information explained part of the genetic variance and was used in the calculation of GEBV of all animals; that is, it was assumed that all males and females of all ages had been genotyped. The proportion of the genetic variance that was explained by simulated marker information $(M)$ varied: $0,20,40,60,80$, or $100 \%$. For $M=0 \%$, genomic information did not explain any variation so GEBV are 
Table 1. Description of breeding scheme before genomic selection

\begin{tabular}{lc}
\hline Item & Value \\
\hline Years before genomic selection, n & 25 \\
Females born per year, n & 1,000 \\
Males born per year, n & 1,000 \\
Cows that obtain a phenotype, n & 1,000 \\
Heritability of cow's phenotype & 0.30 \\
Age when cow's phenotype is available, yr & 3 \\
Age when cows are culled, yr & 6 \\
Bulls that obtain a phenotype, n & 200 \\
Heritability of bull's phenotype & 0.90 \\
Age when bull's phenotype is available, yr & 5 \\
Age when bulls are culled, yr & 8 \\
Dams, n & 200 \\
Minimum age of dams, yr & 3 \\
Progeny per dam & 10 \\
Sires, n & 20 \\
Minimum age of sires, yr & 5 \\
Progeny per sire, n & 100 \\
Genetic variance explained by genetic markers, $\%$ & 0 \\
\hline
\end{tabular}

actually EBV, but will be called GEBV throughout the paper to maintain consistency with results from $M>$ $0 \%$. (2) The minimum possible age to be selected as dam and sire was either 3 and 5 yr (proven; PROV) or 1 and 1 yr (young; YNG), respectively. (3) The number of progeny-tested bulls was reduced from 200 to $100,75,50$, or 25 . (4) To compare the alternative designs at a similar rate of inbreeding, all scenarios were also evaluated with $5,10,15,20,30,40,50,60$, 80,160 , and 200 sires per year to produce the next generation.

Each scenario was replicated 100 times, and the presented results are averages over the 100 replicates.

\section{Simulation of Breeding Values}

True breeding values for 1 total merit index were simulated as

$$
u_{i}=u_{i, M}+u_{i, P}
$$

where $u_{i}$ is the total breeding value for animal $i, u_{i, M}$ is the marker part of the breeding value which can be fully explained by genetic markers and $u_{i, P}$ is the polygenic part of the breeding value which cannot be traced by genetic markers and which was assumed to be independent of $u_{i, M}$. For animals in the base populations, the marker and polygenic breeding values were sampled from a normal distribution: $u_{i, M} \sim \mathrm{N}\left(0, \sigma_{M}^{2}\right)$ and $u_{i, P} \sim \mathrm{N}\left(0,1-\sigma_{M}^{2}\right)$. For subsequent generations, the marker breeding values were simulated as

$$
u_{i, M}=\frac{1}{2} u_{i, M, \text { sire }}+\frac{1}{2} u_{i, M, d a m}+u_{i, M, M S},
$$

where $u_{i, M, \text { sire }}$ and $u_{i, M, d a m}$ are the marker breeding values of the sire and dam of animal $i$, respectively, and $u_{i, M, M S}$ is the marker Mendelian sampling effect, which was drawn from a univariate normal distribution:

$$
u_{i, M, M S} \sim \mathrm{N}\left\{0, \frac{1}{2}\left[1-\frac{1}{2}\left(F_{i, \text { sire }}+F_{i, d a m}\right)\right] \sigma_{M}^{2}\right\}
$$

where $F_{i, s i r e}$ and $F_{i, d a m}$ are the pedigree inbreeding coefficients of the sire and dam of animal $i$ (Meuwissen and Luo, 1992), respectively, and $\sigma_{M}^{2}$ was the genetic variance that could be explained by the markers, which was equal to $0,0.20,0.40,0.60,0.80$, or 1.00 , corresponding to $M=0,20,40,60,80$, and $100 \%$. Note that $M$ is smaller than the reliability of the GEBV, because the GEBV will also include information from phenotypes, as will be explained later.

Similarly, the polygenic breeding values were calculated as

$$
u_{i, P}=\frac{1}{2} u_{i, P, \text { sire }}+\frac{1}{2} u_{i, P, d a m}+u_{i, P, M S},
$$

and the polygenic Mendelian sampling effect was drawn from a univariate normal distribution:

$$
u_{i, P, M S} \sim \mathrm{N}\left\{0, \frac{1}{2}\left[1-\frac{1}{2}\left(F_{i, \text { sire }}+F_{i, \text { dam }}\right)\right]\left[1-\sigma_{M}^{2}\right]\right\},
$$

assuming a genetic variance of 1 in the base population. Note that the effect of selection on the variance of true

Table 2. Description of genomic selection breeding scheme for parameters that differed with the breeding scheme before genomic selection

\begin{tabular}{ll}
\hline Parameter & Value \\
\hline Years with genomic selection, $\mathrm{n}$ & 25 \\
Bulls that obtain a phenotype, $\mathrm{n}$ & $25,50,75,100$, or 200 \\
Minimum age of dams, yr & 1 (young) or 3 yr (proven) \\
Minimum age of sires, yr & 1 (young) or 5 yr (proven) \\
Sires, $\mathrm{n}$ & $5,10,15,20,30,40,50,60,80,160$, or 200 \\
Progeny per sire, $\mathrm{n}$ & $400,200,133,100,67,50,40,33,25,13$, or 10 \\
Genetic variance explained by genetic markers & $M=0,20,40,60,80$ or $100 \%$ \\
\hline
\end{tabular}


breeding values is taken into account via a reduction in the variance of the parent average (Bulmer, 1971). The genetic variance will reduce as a result of inbreeding, but the proportion of the genetic variance that can be explained by genetic markers is kept constant $(M)$.

\section{Simulation of Phenotypes}

Phenotypes were simulated for cows and bulls when they were 3 and 5 yr old, respectively, based on their total breeding value and a residual term:

$$
y_{i}=u_{i}+e_{i}
$$

where $e_{i}$ was drawn from a univariate normal distribution:

$$
e_{i} \sim \mathrm{N}\left(0, \frac{1-h^{2}}{h^{2}}\right),
$$

where $h^{2}=0.3$ for cows and $h^{2}=0.9$ for bulls.

\section{Estimation of Breeding Values}

Before the introduction of genomic selection in year 26 , the marker breeding values were unknown and EBV were estimated from the available phenotypic and pedigree information using a BLUP model: $\mathbf{y}=\mathbf{u}+\mathbf{e}$, where the variance of $\mathbf{u}$ and $\mathbf{e}$ were assumed to be known: $\operatorname{var}(\mathbf{u})=\mathbf{A}$, where $\mathbf{A}$ is the additive genetic relationship matrix derived from the full pedigree, and $\operatorname{var}(\mathbf{e})=I \times \frac{1-h^{2}}{h^{2}}$, where $h^{2}=0.3$ for cows and $h^{2}=$ 0.9 for bulls. The model was solved using iteration on data with a preconditioned conjugate gradient algorithm (Strandén and Lidauer, 1999).

After the introduction of genomic selection in year 26 , the marker breeding values $u_{i, M}$ were assumed to be known for all animals without error, whereas the polygenic breeding values were estimated from the pedigree and phenotypic information:

$$
\hat{\mathbf{u}}=\mathbf{u}_{M}+\hat{\mathbf{u}}_{P}
$$

The polygenic breeding values were estimated with a BLUP model:

$$
\mathbf{y}_{P}=\mathbf{u}_{P}+\mathbf{e},
$$

where $\mathbf{y}_{P}=\mathbf{y}-\mathbf{u}_{M}$. Again, the variance of $\mathbf{u}_{P}$ and $\mathbf{e}$ were assumed to be known:

$$
\operatorname{var}\left(\mathbf{u}_{P}\right)=\mathbf{A} \times\left(1-\sigma_{M}^{2}\right) \text { and } \operatorname{var}(\mathbf{e})=I \times \frac{1-h^{2}}{h^{2}} .
$$

Note that for $M=40 \%$, the reliability of the GEBV for a genotyped young animal whose parents have a phenotype $\left(h^{2}=0.30\right.$ and 0.90$)$ and no other information

equals $0.40+(1-0.40) \times \frac{1}{4}(0.30+0.90)=0.58, \quad$ which approximates the reliabilities of GEBV that have been observed in cross-validation studies when thousands of progeny-tested bulls were used as reference population (VanRaden et al., 2009).

\section{Rate of Genetic Gain and Rate of Inbreeding}

Scenarios were compared by the rate of genetic gain per year, the rate of inbreeding per year, and the rate of inbreeding per generation. The rate of genetic gain per year was calculated as the difference in average true breeding value $(u)$ of the 2,000 animals born in year 50 and the 2,000 animals born in year 25, divided by $25 \mathrm{yr}$. The rate of inbreeding per year was derived from $\Delta F=\frac{F_{t}-F_{t-1}}{1-F_{t-1}}$ (Falconer and Mackay, 1996). If selection has occurred over several years; for example, from year $t_{0}$ to $t$, the rate of inbreeding per year corresponding to that period can be calculated from the average inbreeding coefficients in year $t_{0}$ and $t$ following the equation

$$
\left(1-F_{t}\right)=\left(1-F_{t_{0}}\right)(1-\Delta F)^{t-t_{0}},
$$

which can be rewritten as

$$
\Delta F=1-\left(\frac{1-F_{t}}{1-F_{t_{0}}}\right)^{\frac{1}{t-t_{0}}} .
$$

In our study, the rate of inbreeding per year in the years after the introduction of genomic selection was calculated as

$$
\Delta F=1-\left(\frac{1-F_{50}}{1-F_{25}}\right)^{\frac{1}{25}}
$$

where $F_{25}$ and $F_{50}$ are the average level of inbreeding of the 2,000 animals born in year 25 and year 50, respectively. The rate of inbreeding per generation was 


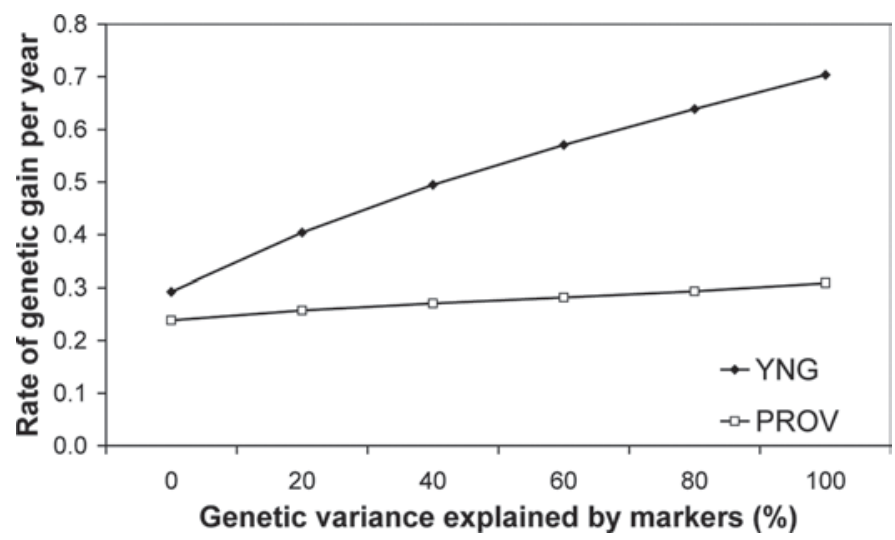

Figure 1. Rate of genetic gain per year (in genetic SD) as a function of the genetic variance explained by markers for scenarios where the minimum age of parents was either 3 and $5 \mathrm{yr}$ (proven; PROV) or 1 and 1 yr (young; YNG) for dams and sires, respectively. All scenarios had 20 sires and 200 progeny-tested bulls per year.

calculated as the rate of inbreeding per year multiplied by the average generation interval of the 2,000 animals born in year 50, where the generation interval was calculated as the average age of the parents at the time of birth of their progeny.

\section{Merit of Young Versus Proven Bulls}

The competitive position of proven bulls compared with young bulls was evaluated each year by the percentage of proven bulls in the top 25 GEBV list in that year, and the difference in average GEBV between the top 25 GEBV young bulls and the top 25 GEBV proven bulls in that year. Bulls were considered to be proven bulls when they had obtained a phenotypic record from their progeny test; that is, when they were 5 yr old. The group of young bulls comprised all bulls between 1 and $4 \mathrm{yr}$ old except those that were culled. Bulls of $0 \mathrm{yr}$ old were not used in the comparison with proven bulls because they cannot reproduce.

\section{RESULTS}

\section{Rate of Genetic Gain and Rate of Inbreeding}

Age of Parents and Genetic Variance Explained by Markers. In the base scenario, where genetic markers explained $0 \%$ of the genetic variation $(M=0 \%)$, dams and sires were at least 3 and 5 yr old at mating, respectively (PROV), 200 bulls were progeny tested per year, and 20 sires per year were used, the rate of genetic gain in true breeding values from year 26 to 50 was 0.238 genetic SD $(\boldsymbol{\sigma})$ per year (Figure 1$)$. Using genetic markers to preselect young bulls before progeny testing increased the rate of genetic gain by up to $30 \%$ to 0.309 $\sigma / \mathrm{yr}(M=100 \%)$. When cows and bulls of $1 \mathrm{yr}$ old were also eligible for selection as parents (YNG), the rate of genetic gain increased by $23 \%$ to $0.292 \sigma / \mathrm{yr}(M$ $=0 \%$. Moving from PROV and $M=0 \%$ to YNG and $M=100 \%$ increased the rate of genetic gain by $195 \%$ to $0.704 \sigma / \mathrm{yr}$.

In the base scenario the rate of inbreeding was $0.18 \%$ per year (Figure 2). With a generation interval of 5.50 yr this corresponds to a rate of inbreeding of $1.00 \%$ per generation (Figure 2). Using genetic markers to preselect young bulls before progeny testing decreased the rate of inbreeding to $0.42 \%$ per generation $(M=$ $100 \%)$. When cows and bulls of 1 yr old were eligible for selection as parents (YNG), the rate of inbreeding was $3.15 \%$ per generation for $M=0 \%$ (generation interval $3.43 \mathrm{yr}$ ), whereas it decreased to $0.63 \%$ per generation for $M=100 \%$ (generation interval $2.12 \mathrm{yr}$ ).

Scenario PROV in combination with $M=0 \%$ was compared with scenario YNG in combination with $M$ $=40 \%$ to assess the effect of using genomic selection with realistic reliabilities of GEBV. In this comparison, the rate of genetic gain increased by $108 \%$ from 0.238 to $0.495 \sigma / \mathrm{yr}$, whereas the rate of inbreeding increased from $1.00 \%$ to $1.14 \%$ per generation and from 0.18 to $0.52 \%$ per year.

Number of Sires. To compare alternative breeding schemes at the same rate of inbreeding per generation, scenarios were evaluated with different numbers of sires per year, varying from 5 to 200. Figure 3 shows the rates of genetic gain corresponding to a rate of inbreeding of $1 \%$ per generation, using linear interpolation between scenarios with different numbers of sires. For PROV, the lowest number of sires that still gave a rate of inbreeding $<1 \%$ per generation was 20 for $M=0 \%$,

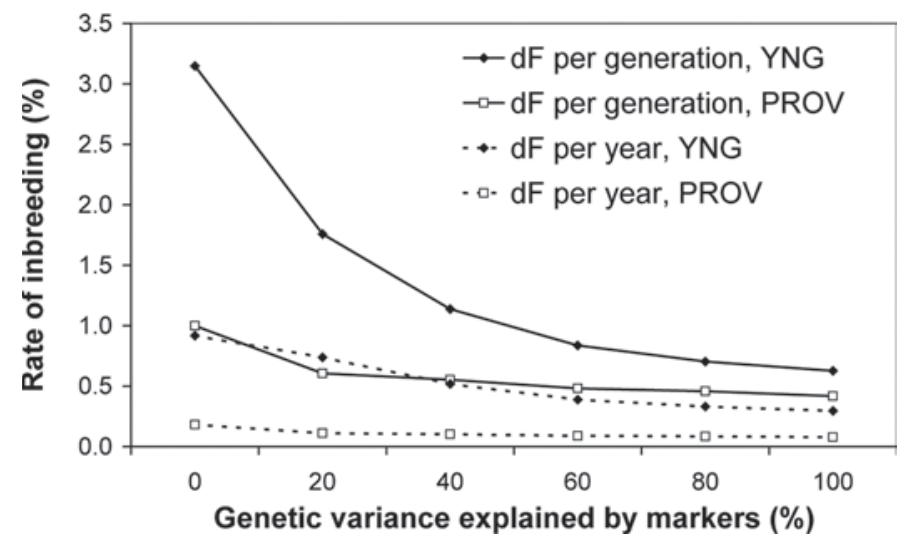

Figure 2. Rate of inbreeding per year and per generation as a function of the genetic variance explained by markers for scenarios where the minimum age of parents was either 3 and $5 \mathrm{yr}$ (proven; PROV) or 1 and 1 yr (young; YNG) for dams and sires, respectively. All scenarios had 20 sires and 200 progeny-tested bulls per year. 


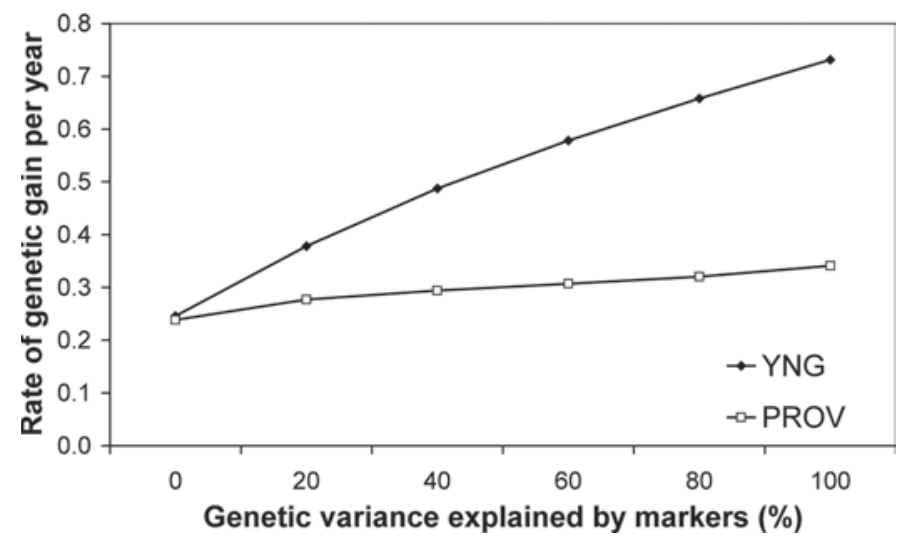

Figure 3. Rate of genetic gain per year (in genetic SD) as a function of the genetic variance explained by markers for scenarios where the minimum age of parents was either 3 and $5 \mathrm{yr}$ (proven; PROV) or 1 and 1 yr (young; YNG) for dams and sires, respectively. The results correspond to a rate of inbreeding of $1 \%$ per generation, using interpolation between scenarios with different numbers of sires. All scenarios had 200 progeny-tested bulls per year.

10 for $M=20$ to $80 \%$, and only 5 for $M=100 \%$. For YNG, however, many more sires per year were needed to keep the rate of inbreeding below $1 \%$ per generation: $>200,60,25,20,15$, and 15 sires per year for $M=$ $0,20,40,60,80$, and $100 \%$, respectively. The rates of genetic gain with a restricted rate of inbreeding in Figure 3 were slightly different from those in Figure 1 , where 20 sires were used across all scenarios. The largest difference in rate of genetic gain was observed for YNG and $M=0 \%$, where the rate of genetic gain decreased by approximately $0.05 \sigma / \mathrm{yr}$.

Number of Progeny-Tested Bulls. When the number of progeny-tested bulls was reduced from 200 to 25 per year, the rate of genetic gain for PROV and $M$ $=0 \%$ reduced from 0.238 to $0.204 \sigma / \mathrm{yr}(-14 \%)$ while the rate of inbreeding increased from 1.00 to $1.65 \%$ per generation (data not shown). For PROV and larger $M$, the effects of progeny testing fewer bulls were smaller, and it had no effect at all for $M=100 \%$. For YNG and $M=0 \%$, progeny testing 25 instead of 200 bulls per year decreased the rate of genetic gain from 0.292 to 0 . $\sigma / \mathrm{yr}(-5 \%)$ while the rate of inbreeding increased from 3.15 to $3.97 \%$ per generation (data not shown). This increase in rate of inbreeding resulted from an increase in the proportion of young bulls as sires. For YNG and $M \geq 20 \%$, reducing the number of progeny-tested bulls had negligible effects on the rate of genetic gain and the rate of inbreeding because almost all sires were young bulls.

Variation in Rate of Genetic Gain. The rates of genetic gain reported above were based on averages over 100 replicates. The variation in the rates of genetic gain between replicates indicates the amount of risk associated with different scenarios. The standard deviation of rate of genetic gain was $0.006 \sigma / \mathrm{yr}$ (mean was $0.238 \sigma / \mathrm{yr}$ ) for the base scenario PROV with $M=0 \%$, which decreased to $0.004 \sigma / \mathrm{yr}$ for $M=100 \%$. YNG had more variation in rate of genetic gain than PROV, with standard deviations equal to 0.015 and $0.007 \sigma / \mathrm{yr}$ for $M=0$ and $100 \%$, respectively. Across scenarios, the variance of the rate of genetic gain followed the rate of inbreeding per year, as expected (Meuwissen, 1991).

\section{Merit of Young Versus Proven Bulls}

Age of Parents and Variance Explained by Markers. In year 50 of the base scenario, the top 25 GEBV list constructed from all bulls of $\geq 1$ yr old, contained $67 \%$ proven bulls; that is, bulls of $\geq 5$ yr old that had obtained a phenotypic record from their progeny test (Figure 4; PROV and $M=0 \%$ ). When genetic markers explained some part of the genetic variance, the proportion of proven bulls in top 25 GEBV list decreased substantially to $29,9,3,1$, and $1 \%$ for $M=$ $20,40,60,80$, and $100 \%$, respectively. For YNG and $M$ $=0 \%, 31 \%$ of the top 25 GEBV list were proven bulls, but the proportion of proven bulls in the top 25 GEBV list reduced rapidly to $0 \%$ for $M \geq 40 \%$. This was also reflected by the average age of the top 25 GEBV bulls, which was $4.11 \mathrm{yr}$ for PROV and $M=0 \%, 2.6 \mathrm{yr}$ for YNG and $M=0 \%, 1.4 \mathrm{yr}$ for PROV and $M=100 \%$, and $1.1 \mathrm{yr}$ for YNG and $M=100 \%$ (data not shown). For YNG and $M \geq 40 \%$, the average age of the top 25 GEBV bulls was $\leq 1.1 \mathrm{yr}$, which indicates that almost all top bulls were 1 yr old.

The average difference between the top 25 GEBV young bulls and the top 25 GEBV proven bulls in year 50 was $-0.19 \sigma$ for PROV and $M=0$ (data not shown), indicating that, in the scenario without genomic selection, the best 25 proven bulls were better than the best 25 young bulls. For YNG and $M=0$, however, the difference was $+0.13 \sigma$ (Figure 5 ); that is, young bulls were better than proven bulls. This difference rapidly increased with increasing $M$ to $1.27 \sigma$ for YNG and $M$ $=40 \%$ and up to $2.74 \sigma$ for YNG and $M=100 \%$. In scenario PROV, the average difference between the top young and proven bulls was 0.51 and $1.21 \sigma$ for $M=40$ and $100 \%$, respectively.

Number of Progeny-Tested Bulls. To determine the optimal number of bulls for progeny testing, the competitive position of proven bulls relative to young bulls needs to be assessed. When fewer bulls were progeny tested, the average GEBV of the top 25 proven bulls was lower because some of the best bulls were not selected for progeny testing based on their GEBV as a young bull. Consequently, the difference between the top 25 GEBV young bulls and the top 25 GEBV 


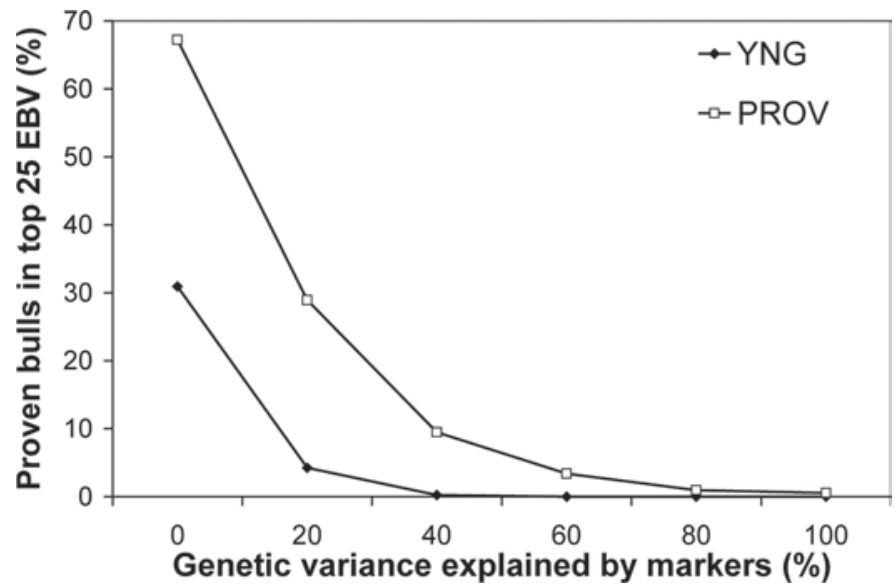

Figure 4. Percentage of proven bulls (age $>5$ yr) in the top 25 genomic EBV list, as a function of the genetic variance explained by markers for scenarios where the minimum age of parents was either 3 and $5 \mathrm{yr}$ (proven; PROV) or 1 and $1 \mathrm{yr}$ (young; YNG) for dams and sires, respectively. All scenarios had 20 sires and 200 progeny-tested bulls per year.

proven bulls increased. For YNG and $M=0 \%$, reducing the number of progeny-tested bulls from 200 to 25 increased this difference from 0.13 to $0.61 \sigma$ (Figure 5). For YNG and $M=40 \%$, the difference between the top young and proven bulls increased from 1.27 to $1.60 \sigma$ when only 25 bulls were progeny tested. The average GEBV of the top 25 proven bulls was reduced by 0.06 , 0.18 and $0.33 \sigma$ when 100,50 or 25 bulls were progeny tested per year. For $M=100 \%$, progeny testing fewer bulls had no effect because the 25 best proven bulls were already identified as young bulls.

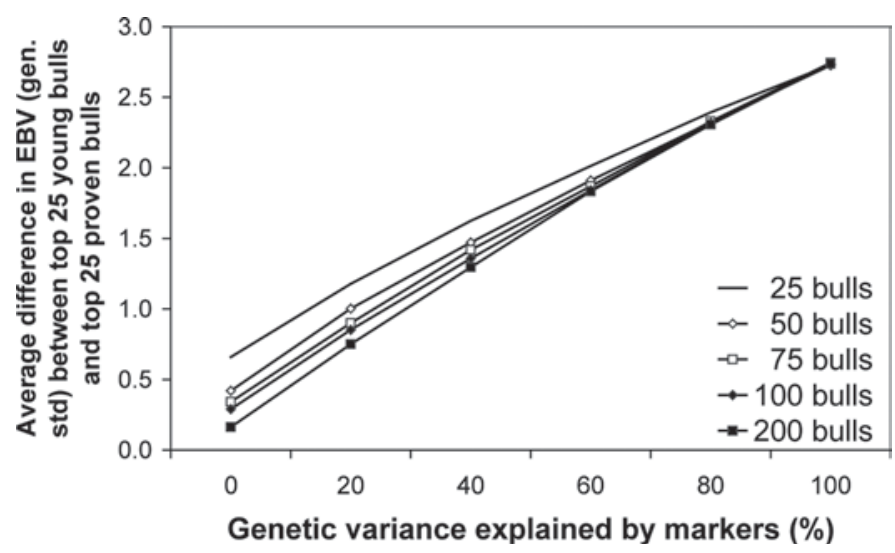

Figure 5. Average difference in genomic EBV (GEBV; in genetic $\mathrm{SD}$ ) between the top 25 GEBV young bulls (ages 1-4 yr) and the top 25 GEBV proven bulls (age $\geq 5 \mathrm{yr}$ ), as a function of the genetic variance explained by markers for scenarios where the number of progenytested bulls was varied between 25 and 200. All scenarios had 20 sires per year, and the minimum age for parents was $1 \mathrm{yr}$. Positive values mean young bulls had higher GEBV than did proven bulls.

\section{DISCUSSION}

\section{Stochastic Simulation}

In this study, the effects of reliability of GEBV, the use of young animals as parents, and the number of progeny-tested bulls on the rate of genetic gain, the rate of inbreeding, and the relative merit of young versus proven bulls were assessed. A closed nucleus breeding scheme was simulated using parameters applicable to dairy cattle. Stochastic simulation was chosen over deterministic methods (Dekkers, 2007) because it allowed us to assess both rate of genetic gain and rate of inbreeding in a 2-stage selection scheme (before and after progeny test) with overlapping generations and a comparison of young and proven bulls in the top GEBV list. Furthermore, effects of selection (Bulmer, 1971) and inbreeding (Keightley and Hill, 1987) on genetic variance can be accounted for much more easily.

\section{Rate of Genetic Gain}

Spelman et al. (1999) derived responses to selection deterministically for 4 paths of selection with overlapping generations in a simulated dairy cattle breeding scheme with 140 progeny-tested bulls, using 3 bull sires and 450 bull dams per year (i.e., fewer bull sires and more bull dams than in our study). Rates of genetic gain for their scenario comparable to $\mathrm{PROV}$ and $M$ $=0 \%$ was $0.258 \sigma / \mathrm{yr}$, which increased to $0.320 \sigma / \mathrm{yr}$ for their scenario with PROV and $M=100 \%$; that is, very consistent with the 0.238 and $0.309 \sigma / \mathrm{yr}$ found in our study (Figure 1). For YNG, Spelman et al. (1999) found a rate of genetic gain of 0.282 and $0.577 \sigma / \mathrm{yr}$ for $M=0$ and $100 \%$, respectively. These values were 0.292 and $0.704 \sigma / \mathrm{yr}$ in our study. The higher rates of genetic gain for YNG and $M=100 \%$ are caused by the intense and accurate selection of dams in our study, whereas Spelman et al. (1999) assumed no selection in the dams for cows pathway. Meuwissen and van Arendonk (1992) modeled a closed nucleus comparable to YNG and observed a rate of genetic gain of 0.297 and $0.412 \sigma / \mathrm{yr}$ for $M=0 \%$ and $25 \%$, respectively, which is consistent with the 0.292 and $0.427 \sigma / y r$ in our study (using linear interpolation for $M=25 \%$ ). The standard deviation of the rate of genetic gain, however, was about 10-fold higher than in our study, possibly because of the smaller size of the nucleus (512 vs. 2000 animals born per year) and smaller number of sires (8 vs. 20). Schrooten et al. (2005) modeled a closed nucleus comparable to PROV and observed that the rate of genetic gain increased by $21 \%$ when $M$ increased from 0 to $50 \%$, whereas this increase was only $16 \%$ in our study (using linear interpolation for $M=50 \%$ ). Marker information was more beneficial in PROV in their study because females were 
preselected before they obtained a phenotype, whereas all females obtained a phenotype in our study. Schrooten et al. (2005) also studied a scenario for $M=50 \%$ and a reduction of the generation interval from 5 to 3 yr by using young bulls as sires. This resulted in a rate of genetic gain that was $68 \%$ higher than the base scenario of PROV and $M=0 \%$. In our study, the generation interval was further reduced in YNG, resulting in a higher rate of genetic gain of $125 \%$ compared with the base scenario of PROV and $M=0 \%$ (using linear interpolation for $M=50 \%$ ).

\section{Rate of Inbreeding}

Rates of inbreeding per generation decreased with increasing $M$, as expected (Daetwyler et al., 2007; Dekkers, 2007). The use of young animals as sires and dams, however, increased the rate of inbreeding per generation substantially, especially for low $M$. This is caused by the lower accuracy of selection when young animals are selected as parents, leading to more weight on parent-average EBV and therefore more co-selection of sibs. Rate of inbreeding per year was much higher for YNG than PROV across all $M$, because of the shorter generation interval. The biological risks of inbreeding, such as genetic variance reduction, inbreeding depression, and accumulation of deleterious alleles, however, are more associated with inbreeding rate per generation, rather than per year, because processes that may compensate for inbreeding, such as mutation, also occur per generation (Villanueva et al., 2000). An argument to consider the rate of inbreeding per year in addition to the rate of inbreeding per generation is that inbreeding depression, homozygosity for deleterious alleles, and reduction in genetic variance are associated with the level of inbreeding and will therefore occur sooner if the rate of inbreeding per year is higher (Villanueva et al., 2000).

Comparing alternative breeding schemes is optimally done at the same rate of inbreeding (Quinton et al., 1992). To achieve this, the number of sires was adjusted such that the rate of inbreeding was just below $1 \%$ per generation (Figure 3). This had only small effects on the rates of genetic gain compared with the scenarios without restriction on the rate of inbreeding (Figure 1). The largest effects were observed for YNG and $M \leq$ $20 \%$, where the rate of genetic gain decreased by about $0.04 \mathrm{\sigma} / \mathrm{yr}$ (Figure 3 vs. Figure 1). If the rate of inbreeding were restricted to a fixed rate per year, rather than per generation, the required number of sires for YNG would need to be increased substantially, leading to a lower rate of genetic gain. For example, if the rate of inbreeding of PROV and $M=0 \%$ were taken as standard, the rate of genetic gain for YNG and $M=40 \%$ would be $0.488 \sigma / \mathrm{yr}$ when the restriction was on the rate of inbreeding per generation $(1.00 \%)$ and $0.425 \sigma /$ yr when the restriction was on the rate of inbreeding per year $(0.18 \%)$.

In this study we assumed that genetic variance decreased with the inbreeding level based on pedigree. This may not be valid if a small number of QTL explain a large part of the genetic variance, because then selection on the markers would lead to more inbreeding than what is observed from pedigree, or even fixation at the QTL (Pedersen et al., 2009). Another assumption was that the genetic markers explained a constant part of the genetic variation that was generated every year $(M)$. This implies that no reduction in accuracy occurs due to decay of linkage disequilibrium between markers and QTL. This may not be valid if the reference population is established once and never updated, but in practice it seems likely that reference populations will be updated continuously. Furthermore, it may be expected that continued research and development of DNA technology and statistical tools and the addition of more bulls and cows to reference populations will increase rather than decrease reliabilities over time.

\section{Number of Progeny-Tested Bulls}

Our study was consistent with Schrooten et al. (2005) who observed that, in a selection scheme where proven bulls and cows are used as parents and genetic markers explained $20 \%$ of the genetic variance, the number of progeny-tested bulls could be reduced from 200 to 50 while maintaining the same rate of genetic gain. When young animals were used as parents, however, progeny testing fewer bulls had no influence on the rate of genetic gain or the rate of inbreeding, which was also observed by Spelman et al. (1999). Breeding companies need to predict the demand for proven bull semen compared with young bull semen in a situation with a clear superiority in GEBV of young bulls over proven bulls. Some farmers may still favor proven bulls with high reliability but it is expected that a large group of farmers will switch to using young bulls. If the market for proven bull semen becomes smaller, the returns on investments from progeny testing become smaller as well. Reducing the number of progeny-tested bulls is an option to cut costs, but at the cost of a reduction in average GEBV of the top proven bulls. When the reliability of GEBV is high, however, this cost is small because the best young bulls can be accurately selected before progeny testing. An argument to continue progeny testing a large number of bulls might be to update the reference population in the future. It is expected, however, that it is more cost effective to update the reference population by genotyping cows. 


\section{CONCLUSIONS}

This stochastic simulation study shows that genomic selection will increase the rate of genetic gain and decrease the rate of inbreeding per generation. The effects of genomic selection were, at maximum, $+30 \%$ of genetic gain per year when the generation interval was kept constant to traditional progeny-test schemes. Using young animals without their own or progeny performance information as parents doubled the rate of genetic gain for intermediate reliabilities of GEBV, whereas the rate of inbreeding per generation was the same as in a traditional BLUP selection scheme. The rate of inbreeding per year, however, increased more than 2-fold because of the reduction in generation interval. Because of the higher rates of genetic gain per year and higher reliabilities of GEBV, young bulls dominated top GEBV rankings and had $>1 \sigma$ higher GEBV than the top proven bulls. As a result, progeny testing fewer bulls had negligible effects on the rate of genetic gain or the rate of inbreeding. Furthermore, the quality of the proven bull team was maintained as well because of the more accurate selection of young bulls before progeny testing.

\section{ACKNOWLEDGMENTS}

The authors are grateful for discussions with Piter Bijma, Henk Bovenhuis, Henri Heuven, Mario Calus, Han Mulder, John Bastiaansen, and Albart Coster (all of Animal Breeding and Genomics Centre, Wageningen University, Wageningen, the Netherlands) on the work presented here.

\section{REFERENCES}

Bulmer, M. G. 1971. The effect of selection on genetic variability. Am. Nat. 105:201-211.

Daetwyler, H. D., B. Villanueva, P. Bijma, and J. A. Woolliams. 2007. Inbreeding in genome-wide selection. J. Anim. Breed. Genet. 124:369-376.

Dekkers, J. C. M. 2007. Prediction of response to marker-assisted and genomic selection using selection index theory. J. Anim. Breed. Genet. 124:331-341.
Falconer, D. S., and T. F. C. Mackay. 1996. Introduction to Quantitative Genetics. 4th ed. Longman, Harlow, UK.

Hayes, B. J., P. J. Bowman, A. J. Chamberlain, and M. E. Goddard. 2009. Invited review: Genomic selection in dairy cattle: Progress and challenges. J. Dairy Sci. 92:433-443.

Kashi, Y., E. Hallerman, and M. Soller. 1990. Marker-assisted selection of candidate bulls for progeny testing programmes. Anim. Prod. 51:63-74.

Keightley, P. D., and W. G. Hill. 1987. Directional selection and variation in finite populations. Genetics 117:573-582.

Mackinnon, M. J., and M. A. J. Georges. 1998. Marker-assisted preselection of young dairy sires prior to progeny testing. Livest. Prod. Sci. 54:229-250.

Meuwissen, T. H. E. 1991. Expectation and variance of genetic gain in open and closed nucleus and progeny testing schemes. Anim. Prod. 53:133-141.

Meuwissen, T. H. E., B. J. Hayes, and M. E. Goddard. 2001. Prediction of total genetic value using genome-wide dense marker maps. Genetics 157:1819-1829.

Meuwissen, T. H. E., and Z. Luo. 1992. Computing inbreeding coefficients in large populations. Genet. Sel. Evol. 24:305-313.

Meuwissen, T. H. E., and J. A. M. van Arendonk. 1992. Potential improvements in rate of genetic gain from marker-assisted selection in dairy cattle breeding schemes. J. Dairy Sci. 75:1651-1659.

Pedersen, L. D., A. C. Sørensen, and P. Berg. 2009. Marker-assisted selection can reduce true as well as pedigree-estimated inbreeding. J. Dairy Sci. 92:2214-2223.

Quinton, M., C. Smith, and M. E. Goddard. 1992. Comparison of selection methods at the same level of inbreeding. J. Anim. Sci. 70:1060-1067.

Schaeffer, L. R. 2006. Strategy for applying genome-wide selection in dairy cattle. J. Anim. Breed. Genet. 123:218-223.

Schrooten, C., H. Bovenhuis, J. A. M. van Arendonk, and P. Bijma. 2005. Genetic progress in multistage dairy cattle breeding schemes using genetic markers. J. Dairy Sci. 88:1569-1581.

Smith, C. 1967. Improvement of metric traits through specific genetic loci. Anim. Prod. 9:349-358.

Soller, M., and J. S. Beckmann. 1983. Genetic polymorphism in varietal identification and genetic improvement. Theor. Appl. Genet. 67:25-33.

Spelman, R. J., D. J. Garrick, and J. A. M. van Arendonk. 1999. Utilisation of genetic variation by marker assisted selection in commercial dairy cattle populations. Livest. Prod. Sci. 59:51-60.

Strandén, I., and M. Lidauer. 1999. Solving large mixed linear models using preconditioned conjugate gradient iteration. J. Dairy Sci. 82:2779-2787.

VanRaden, P. M., C. P. VanTassel, G. R. Wiggans, T. S. Sonstegard, R. D. Schnabel, J. F. Taylor, and F. S. Schenkel. 2009. Invited review: Reliability of genomic predictions for North American Holstein bulls. J. Dairy Sci. 92:16-24.

Villanueva, B., P. Bijma, and J. A. Woolliams. 2000. Optimal mass selection policies for schemes with overlapping generations and restricted inbreeding. Genet. Sel. Evol. 32:339-355. 